Monitoring the patient with carotid disease for cerebral ischaemia is a challenge to the anesthetist. The best monitor of decreased cerebral blood flow is a change in mental status, but not every surgical procedure can be performed with the patient awake. Even if the procedure is performed using a regional anaesthetic technique, a patient may require sedation thus making it difficult to monitor cerebral function. When EEG is used to monitor for cerebral ischaemia during general anaesthesia, the following guidelines should be met:

First, a 16 lead EEG monitor should be used as the processed EEG generated with a two or four lead EEG system may yield misleading information. Second, someone trained in the application and interpretation of the EEG should be present throughout the procedure. Third, the anaesthetic technique must be tightly controlled so that the agents used do not interfere with the EEG tracing. Fourth, a clinical manoeuvre to correct abnormal findings from the EEG must be available to improve blood flow to the brain.

When anaesthesia is administered to a patient with $T A$, the maintenance of blood pressure to avoid cerebral ischaemia from hypotension or rupture of an aneurism from hypertension is the priority. This can be accomplished by a variety of anaesthetic techniques and should be individualized based upon the patient's clinical status and the anaesthesiologist's experience. Monitoring for cerebral ischaemia, EEG versus the awake patient's mental status, should also be individualized depending on physician experience.

Yaakov Beilin MD

Howard Bernstein MD

Department of Anesthesiology

The Mount Sinai Medical Center

1 Gustave Levy Place, Box 1010

New York, NY 10029, USA

REFERENCE

1 Beilin Y, Bernstein H. Successful epidural anaesthesia for a patient with Takayasu's arteritis presenting for Caesarean section. Can J Anaesth 1993; 40: 64-6.

\section{Perforation of the trachea by a central venous catheter guidewire}

To the Editor:

An 81-yr-old man was admitted for elective repair of an abdominal aortic aneurysm. Preoperative assessment was consistent with abdominal aortic aneurysm but there were no other important findings, and the neck anatomy appeared normal. For intraoperative monitoring a 16 gauge "Leadercath" central venous catheter was carefully checked, including the guidewire $(1.1 \mathrm{~mm}$ wide, $46 \mathrm{cms}$ long) which was straight, soft-tipped with a smooth round end with no sharp edges. The right internal jugular vein was located easily at a depth of $1.5 \mathrm{~cm}$, but the guidewire met with resistance, and needle and guidewire were removed together. The vein was found easily again, the guidewire advanced cautiously, and slight resistance was encountered at the same depth; increased but not excessive force led to loss of resistance, and the wire advanced smoothly. The catheter was threaded over the guidewire easily but on removal of the guidewire fresh blood appeared suddenly in the endotracheal tube. A haematoma developed and local pressure caused this to disappear rapidly, but the simultaneous reappearance of further fresh blood in the endotracheal tube made it obvious that the haematoma was draining into the trachea. The guidewire revealed two kinks near the tip. Aspiration of the catheter generated a negative pressure in the syringe but neither blood nor air was obtained, indicating that it was extravascular, but had not entered the trachea properly because of the trachea's intrinsic rigidity. Slight resistance is not uncommon during guidewire insertion, but the use of firm but not excessive pressure resulted in the guidewire perforating the trachea. Central venous access was secured by another route and the procedure continued uneventfully; A chest radiograph and bronchoscopy confirmed blood in the right upper lobe. No tracheal injury was visualized because of blood and clots obscuring the tracheal mucosa. The lungs were ventilated overnight and the trachea was extubated the next morning; a radiograph showed clearing of the previous findings and further recovery was uneventful.

Many complications of central venous catheterization caused by the needle have been described. ${ }^{1}$ Guidewires techniques, while popular, have their own complications such as fragmentation and embolisation, ${ }^{2,3}$ retention, ${ }^{4}$ uncoiling, ${ }^{5,6}$ and knotting. ${ }^{7}$ One report documents venous damage leading to massive haemorrhage, ${ }^{6}$ and another describes the new onset of complete heart block, ${ }^{8}$ both caused by guidewires. Tracheal perforation by a straighttipped guidewire, an occurrence that is not previously described in the literature serves to emphasise the potential for soft-tissue damage during invasive procedures and the authors recommend the use of soft J-tipped guidewires to avoid this complication.

V. Dimitriou MD DEAA

A.M. O'Leary FCAnaes

Department of Anaesthesia

University Hospital, Queens Medical Centre

Nottingham. NG7 2UH England

Present address:

Dr. A.M. O'Leary

Department of Anesthesiology

Albany Medical Center

New Scotland Avenue

New York 12208 USA 


\section{REFERENCES}

1 Breen MT, Kageler $W V$. Puncture of the trachea during cathetenization of the subclavian vein (Letter). $\mathbf{N}$ Engl $\mathbf{J}$ Med 1989; Apr 27; 320: 1148.

2 Barker P. Guidewire embolism (Letter). Anaesthesia 1991; 46: 595.

3 Schwartz AJ, Horrow JC, Jobes DR, Ellison N. Guide wires-a caution. Crit Care Med 1981; 9: 347.

4 Panos $A L$, Common AA, Salerno TA. Unusual complication of $\mathrm{J}$ wire used for central venous cannulation (Letter). Ann Thorac Surg 1991; 52: 581-2.

5 Hickman $J A, M c$ Crirrick $A$. Failure of a spring guide wire (Letter). Anaesth Intensive Care 1990; 18: 587.

6 Chacko J, Litz S, Morrison J. Complications with guide wires for central venous cannulation (Letter). Can J Anaesth 1991; 38: 258-9.

7 Gravenstein JS, Paulus DA. Clinical Monitoring Practice. 2nd ed, Philadelphia: Lippincott, 1987; 105-56.

8 Eissa NT, Kvetan V. Guide wire as a cause of complete heart block in patients with pre-existing left bundle branch block. Anesthesiology 1990; 73: 772-4.

\section{Dental restoration using oral ketamine}

To the Editor:

In recent months, several reports have appeared ${ }^{1,2}$ concerning the use of oral ketamine in mentally handicapped young adults requiring dental treatment. The following case illustrates a recent experience with this technique.

A 20-yr-old man with cerebral palsy and a mental age of eight was scheduled for dental restorations under general anaesthesia. His weight was $100 \mathrm{~kg}$. An attempt to perform the procedure one month earlier had been abandoned. He arrived at the hospital one hour before his scheduled surgery. He drank $40 \mathrm{ml}$ of a mixture of grape juice, sugar and $1000 \mathrm{mg}$ ketamine. He sat in a chair for $10 \mathrm{~min}$ and after 30 minutes, an $i v$ was started and oxygen applied. At $45 \mathrm{~min}$ he was transferred to the operating room. Induction of anaesthesia was completed with propofol and tracheal intubation was facilitated with succinylcholine. He was suctioned for secretions prior to intubation. Anaesthesia was maintained with isoflurane, nitrous oxide/oxygen and morphine. Isoflurane was discontinued $30 \mathrm{~min}$ before completion and a propofol infusion was instituted in an attempt to facilitate early awakening. At several times during the procedure, desaturation was noted each time associated with inspiratory and expiratory wheezes and increased airway pressures. Thick white secretions were suctioned from the endotracheal tube and inhaled salbutamol was used to restore oxygenation and decrease airway pressures. The dental restoration took $150 \mathrm{~min}$. The patient awoke promptly upon discontinuation of anaesthesia, $240 \mathrm{~min}$ after the oral ketamine. No post op desaturation was noted.

The use of oral ketamine in the mentally retarded patient is not new. ${ }^{1,2}$ Previous clinical reports have attested to its safety and effectiveness. ${ }^{3}$ Theoretical considerations for deciding on the appropriate dose have been described by Grant $e t$ al. ${ }^{4}$ We doubt that the relative ease of this man's anaesthetic course could have been successfully duplicated with another agent.

The concern about secretions both oral and endobronchial is important. One previous report describes excessive salivation during emergence as a problem in a child given a higher dose of oral ketamine. ${ }^{5}$ This patient had increased secretions at time of induction and continued to produce secretions during the case. His history of smoking and a prolonged induction with a medication which is known to increase respiratory secretions would have contributed to the secretions which were apparent during the procedure.

Given the circumstances, the outcome was satisfactory and would only be modified by the use of an antisaligogue during the procedure.

Saifee Rashiq MD

Craig Guenther MD

University of Alberta Hospital

Edmonton, Alberta

\section{REFERENCES}

1 Gutstein HB, Johnson KL, Heard MB, Gregory GA. Oral ketamine preanesthetic medication in children. Anesthesiology 1992; 76: 28-33.

2 Petros $A J$. Oral ketamine. Its use for mentally retarded adults requiring day care dental treatment. Anaesthesia 1991; 46: 646-7.

3 Bragg $C L$, Miller BR. Oral ketamine facilitates induction in a combative mentally retarded patient. $\mathbf{J}$ Clin Anaesth 1990; 2: 121-2.

4 Grant IS, Nimmo WS, Clements JA. Pharmacokinetics and analgesic effects of i.m. and oral ketamine. $\mathrm{Br} \mathrm{J}$ Anaesth 1981; 53: 805-10.

5 Hain WR. Oral ketamine (Letter). Anaesthesia 1983; 38: $810-1$. 